\title{
IX. DAS »VOLK«, DER KÖNIG UND SEINE AMTSTRÄGER ZUSAMMENFASSUNG
}

Betrachtet man die in den einzelnen Kapiteln untersuchten Elemente, die die französische Politik in den Trois-Évêchés während der knapp 100 Jahre zwischen der Besetzung im Jahr 1552 und dem Westfälischen Frieden bestimmten, so lassen sich gerade aus der Zusammenschau der methodisch auf sehr unterschiedlichem Wege gewonnenen Erkenntnisse einige Leitlinien erkennen, die im folgenden zusammengefaßt werden sollen. Hierbei zeigt sich auch der Vorteil des gewählten Vorgehens gegenüber einer isolierten Betrachtung beispielsweise des Justizhandelns oder der Justiznutzung, beziehungsweise der Diskurse um juristische Fragen oder rein prosopographischer Ansätze.

Der Herrschaftswechsel in den Trois-Évêchés war mit der Besetzung im Jahre 1552 zunächst weder intendiert, noch gar vollendet. Die Besetzung paßte zwar folgerichtig zur Politik Frankreichs sowohl gegenüber dem Reich als auch gegenüber den lothringischen Territorien. Sie diente jedoch zunächst erst einmal der Grenzsicherung und war nicht von Anfang an auf eine vollständige Eingliederung gerichtet. Diese vollzog sich vielmehr als ein allmählicher Prozeß im Laufe von fast 100 Jahren. Dementsprechend wurde zunächst kaum planmäßig vorgegangen. Dies zeigt sich beispielsweise an der unterschiedlichen und zunächst sich nur allmählich etablierenden Präsenz königlicher Institutionen und Amtsträger in den einzelnen Städten und Territorien. So wurde in den ersten Jahren nach der Besetzung zwar großer Wert auf die Durchsetzung des président gegenüber den städtischen Institutionen in Metz gelegt, von solchen Befugnissen in Toul und Verdun, geschweige denn in den bischöflichen Territorien war jedoch bis zur Jahrhundertwende keine Rede.

Die unter Heinrich IV. zu beobachtende Intensivierung der Bemühungen um eine vollständige Integration der Trois-Évêchés ins französische Königreich brachte ebenfalls zunächst keine umfassende Angleichung. Besonders deutlich wird dies am Beispiel des Hochstifts Metz, das selbst während (oder vielmehr gerade wegen) der Verwaltung durch einen Sohn des Königs seine Unabhängigkeit bis 1631 bewahren konnte.

Insgesamt waren die Bemühungen um eine verstärkte Kontrolle bis zur Errichtung eines königlichen Parlamentsgerichtes in Metz 1633 eher ein Reagieren auf bestimmte Ereignisse und Erfordernisse, die meist primär militärischer Art waren. Ein gezieltes und erfolgreiches Agieren im Sinne einer Vereinheitlichung der Verwaltung, einer umfassenden Kontrolle der Bevölkerung und ihrer Institutionen oder gar einer Zentralisierung kann hier entsprechend nicht beobachtet werden. 
Dennoch existierten entsprechende Vorstellungen zur vollständigen Eingliederung spätestens seit der Regierungszeit Heinrichs IV., wie gerade die Analyse der Vorschläge zur Errichtung eines Parlaments zeigt. Denn mit diesem Gericht wurde eine Institution geschaffen, die für die gesamten Trois-Évêchés zuständig war, und die nicht mehr auf militärische, sondern auf zivile Erfordernisse reagierte. Wie beispielsweise der Auftrag zur Erstellung einer einheitlichen Rechtsgrundlage an die ersten Bediensteten des Gerichts zeigt, wurde nun auch der Versuch einer gewissen Vereinheitlichung unternommen. Gerade der Weg zu einem Parlament macht aber auch deutlich, daß die jeweilige Intensität der Bemühungen um eine vollständige Integration der Trois-Évêchés immer auch von der jeweiligen Machtposition des französischen Königs abhängig war. Auch deshalb wurden gerade unter Heinrich IV. und Ludwig XIII. entscheidende Schritte unternommen.

Bereits vor der Besetzung existierte in den Trois-Évêchés eine deutliche Orientierung nach Frankreich, die den Herrschaftswechsel erleichtert und befördert hat. Hinweise hierfür sind neben der gemeinsamen Sprache auch die Ähnlichkeiten in der Gerichtsorganisation auf unterer Ebene und im Recht der coutume. Auch die politischen Verhältnisse, vor allem die Erstarrung der patrizischen Stadtregimente in Verdun und Metz und das gleichzeitige Streben der Bischöfe nach mehr Kontrolle in ihren Kathedralstädten, haben das Eingreifen Frankreichs und dessen Akzeptanz begünstigt. Zunächst erwies sich so die Besetzung, ähnlich wie es Horst Carl für Reichsterritorien am Niederrhein zusammengefaßt hat, als Testfall für innere Machtverhältnisse und insbesondere in Metz auch als ideale Entfaltungsmöglichkeit für die verstärkt agierenden Ständeversammlungen'.

Die mittelalterliche Verfaßtheit der drei Städte und der geringe staatliche Organisationsgrad der bischöflichen Territorien vor den Ereignissen von 1552 ermöglicht, am Fallbeispiel der Trois-Évêchés die Etablierung moderner Staatlichkeit über den Aufbau entsprechender Institutionen zu beobachten. Die Verknüpfung von Staatsbildung und Herrschaftswechsel ${ }^{2}$ geht hier vor allem von den Institutionen der Gerichtsbarkeit aus und gipfelt in der Errichtung und der Tätigkeit des Parlaments von Metz. Weiterhin war offenbar gerade das neuerworbene Gebiet in Lothringen eine gute "Testmöglichkeit" für neue Institutionen oder Ämter. Dies gilt vor allem für die Funktionen des président

\footnotetext{
${ }^{1}$ Vgl. CARL, Französische Besatzungsherrschaft.

${ }^{2}$ Vgl. dazu die Erläuterungen in der Einleitung sowie den Überblick bei REINHARD, Geschichte der Staatsgewalt, hier S. 125-140 und GENET, La genèse de l'État moderne, in: Actes de la Recherche en sciences sociales 118 (1997) S. 3-18.
} 
und des Intendanten, für die in den Trois-Évêchés neue Kompetenzen etabliert wurden, bevor dies in ganz Frankreich übernommen wurde ${ }^{3}$.

Warum aber dauerte es so lange, bis sich französische Institutionen tatsächlich dauerhaft in den Trois-Évêchés etablieren konnten? Rücksichten auf die außenpolitische Situation waren zwar, wie vor allem in Kapitel IV und VIII gezeigt, durchaus vorhanden, ein konkretes Bestreben des Reiches nach Restitution der drei Städte und Stifte ist jedoch während des gesamten Untersuchungszeitraumes kaum zu beobachten. Wichtiger war hier ein anderer Faktor, der politisches Handeln immer begleitet. Hier aber ist er wegen der sich durch den Herrschaftswechsel ergebenden außen- wie innenpolitischen Rücksichten besonders deutlich zu beobachten. Gemeint ist damit die Interaktion und die Kommunikation zwischen den einzelnen Beteiligten, die in der vorliegenden Arbeit vor allem im Diskurs um die französische Rechtspolitik untersucht wurde. Im gesamten Untersuchungszeitraum ist festzustellen, daß in großem Umfang historische Rechtstitel und Dokumente gesucht wurden, um die französische Politik nach innen wie außen zu legitimieren. Allmählich wurde dann die Rechtsgrundlage der Protektion durch die Konzeption einer auf historische Rechte begründeten Souveränität abgelöst. Das Bemühen um Legitimation der königlichen Herrschaft auch gegenüber der Bevölkerung der Trois-Évêchés zeigt zudem, daß deren Zustimmung zur Entfaltung dieser Herrschaft unabdingbar erschien. Besonders deutlich wird dies in den zahlreichen Denkschriften, die sich damit befaßten, wie die Zuneigung des Volkes (ausgedrückt im Bild der »Herzen«) zu gewinnen sei. Ähnlich sind aber auch beispielsweise die Besuche des Monarchen in den Städten zu werten, bei denen eine direkte Bindung zwischen Herrscher und Bevölkerung hergestellt werden sollte ${ }^{4}$.

Dieses Bemühen um Konsens und Legitimität prägte auch die Politik vor Ort und machte diese nicht selten zahnlos. Zudem taten sich die königlichen Amtsträger, die ihrerseits diese Politik umsetzen sollten, mit der »Eroberung der Herzen « häufig schwer. Das lag oft auch am persönlichen Hintergrund dieser Männer: Vor der Parlamentserrichtung hatten vor allem konfessionelle Unterschiede (mehrere présidents waren Protestanten) und - bei den aus der Region stammenden Amtsträgern - Eifersüchteleien sowie Konflikte mit den Mitbürgern die Akzeptanz bei der Bevölkerung beeinträchtigt. Waren die Amtsträger hingegen um einen Ausgleich mit den angestammten Institutionen bemüht und hierin erfolgreich, konnte dies, wie im Falle des Présidial-Gerichts

${ }^{3} \mathrm{Zu}$ den Intendanten vgl. Anette SMEDLEY-WEILL, Les intendants de Louis XIV, Paris 1995; Roland MOUSNIER, État et commissaire. Recherches sur la création des intendants des provinces (1634-1648), in: Forschungen zu Staat und Verfassung, Festgabe für Fritz Hartung, Berlin 1958, S. 325-344.

${ }^{4} \mathrm{Vgl}$. zu den Besuchen des Königs in französischen Städten Lawrence M. BRYANT, The King and the City in the Parisian Royal Entry Ceremony, Genf 1986. 
unter Charpentier in Metz zu einer quasi Auflösung der königlichen in der alten städtischen Institution führen. Vor diesem Hintergrund sind auch die hier zu beobachtenden Anfänge des Intendantenwesens durchaus differenziert zu sehen, waren diese Amtsträger doch in den Trois-Évêchés eher Männer des Ausgleichs denn "Zentralisierer«.

Erst das Parlament war auch personell in der Lage, seine Gerichtsbarkeit auch gegen Widerstände durchzusetzen, wobei jedoch auch hier eher vorsichtig vorgegangen wurde. Dies zeigt beispielsweise der Umgang mit Urteilen des alten städtischen Gerichts von Metz, der treize, in den ersten Verfahren des Parlaments. Das Extrembeispiel der Hexenprozesse zeigt hierbei, daß gerade die Gerichte im ländlichen Raum noch über Jahrzehnte hinweg die Parlamentszuständigkeiten und -anordnungen erfolgreich ignorieren konnten. Abgesehen von der spektakulären Hinrichtung des Richelieu-Attentäters Alpheston gilt diese Beobachtung auch für Majestätsverbrechen. Diese Exekution muß wie die prächtige Eröffnungszeremonie als eine Art "Öffentlichkeitsarbeit« der neuen Institution gewertet werden ${ }^{5}$. Nicht zuletzt durch die Ortswahl des alten städtischen Richtplatzes in Metz wurde bei dieser Hinrichtung demonstriert, da $ß$ allein das Parlament in Zukunft fuir Strafverfahren zuständig war, und da $B$ es gedachte, hart gegen Widerstand vorzugehen. Dort aber, wo keine konkrete Gefahr zu erwarten war, etwa bei ungehörigen Reden, schien man sich eher nach der in anderen Zusammenhängen immer wieder geäußerten Vorgabe des doucement zu richten ${ }^{6}$. Insofern decken sich die Beobachtungen mit den Ergebnissen Robert Muchembleds zu FranzösischFlandern. Dort hat dieser ebenfalls die Durchsetzung der königlichen Gerichtsbarkeit mit einem Zusammenspiel spektakulärer Hinrichtungen und Gnadenerlasse erklärt ${ }^{7}$. Betrachtet man das Wirken der königlichen Institutionen in Metz, Toul und Verdun bis zur Mitte des 17. Jahrhunderts, liegt zusammenfassend der Schluß nahe, daß »Gesetze die nicht durchgesetzt

\footnotetext{
s Ähnlich MuCHembLed, Temps des supplices, und FouCAulT, Überwachen und Strafen. GESTRICH, Absolutismus, sieht Hinrichtungen hingegen nicht zu Unrecht auch als problematische Situation für die Autorität des Herrschers, vgl. ebd. S. 120-125.

${ }^{6} \mathrm{Zu}$ ähnlichen Ergebnissen kommt für Württemberg Helga SCHNABEL-SCHÜLE, Das Majestätsverbrechen als Herrschaftsschutz und Herrschaftskritik, in: Aufklärung 7 (1994) S. 29-47.

${ }^{7}$ MuChembled, Temps des supplices. Dieser hat allerdings, anders als vorliegende Studie, Hexenprozesse selbst, und nicht ihre Unterdrückung, als Mittel zur Kontrolle des splatten Landes« beobachtet.
} 
wurden ${ }^{8}$ auch in den Trois-Évêchés durchaus mit einem grundsätzlichen »Erfolg« des Staates einhergehen konnten?.

Gerade die parallele Betrachtung von Gerichtsverfahren, Chroniken und Briefquellen vermittelt zudem einen guten Eindruck davon, daß die französische Politik in den Trois-Évêchés grundsätzlich kaum mit Widerstand konfrontiert wurde. Betrachtet man beispielsweise die Anzahl der Verfahren, die das Parlament von Metz wegen Widerstandsäußerungen und ähnlicher Delikte einleitete, erscheint die Bevölkerung nicht zum Aufruhr gestimmt. Ebenso zeigt der Blick in die Tagebücher, Chroniken und Aufzeichnungen, daß der Herrschaftswechsel grundsätzlich kaum in Frage gestellt oder gar abgelehnt wurde. Grundsätzlich schien bei der Masse der Bevölkerung eine eher gleichgültige Sicht des Herrschaftswechsels vorgeherrscht zu haben. Wenn Kritik formuliert wurde, etwa durch die cahiers, wurde vor allem das »Wie« und hier vor allem das Verhalten der königlichen Amtsträger kritisiert. Bemerkenswert ist dabei, daß diese meist nur unter dem konkreten Druck anstehender (von Seiten der königlichen Verwaltung angeordneter) Veränderungen formuliert wurden. Daß keinerlei Unruhen oder gar Aufstände in den Trois-Évêchés zu beobachten sind, ist vor allem dann interessant, wenn man diesen Befund mit der allgemeinen Situation in Frankreich kontrastiert ${ }^{10}$.

Ein gänzlich anderes Bild scheint sich zunächst in den Schreiben der königlichen Amtsträger über die Stimmung der Bewohner zu präsentieren. Diese vermitteln durch den gesamten Untersuchungszeitraum ein eher düsteres Bild von der Situation in den Trois-Évêchés. Die Beschreibung der eigenen Situati-

${ }^{8}$ Mit dieser Formulierung hat Jürgen Schlumbohm auf prononcierte Art den Blick auf das entsprechende Phänomen gelenkt, vgl. Jürgen SCHLuMBOHM, Gesetze, die nicht durchgesetzt wurden - ein Strukturmerkmal des frühmodernen Staates? in: Geschichte und Gesellschaft 23 (1997) S. 647-663.

${ }^{9}$ LANDWEHR, Normdurchsetzung. Landwehr verweist zu Recht auf die Anregungen Anthony Giddens, dessen Thesen auch in der vorliegenden Arbeit die Fragestellung begleiteten. Vgl. Anthony GIDDENS, Die Konstitution der Gesellschaft. Grundzüge einer Theorie der Strukturierung, Frankfurt a. M., New York 1995.

${ }^{10}$ Gerade die ältere Forschung hat sich sehr intensiv mit den Unruhen befaßt, die mit der Durchsetzung absolutistischer Herrschaftsinteressen häufig einherzugehen schienen: Seit den sechziger Jahren wurden um die Bewertung der zahlreichen Revolten des Ancien Régime heftige Debatten geführt, vgl. nur Boris F. PORSCHNEW, Die Volksaufstände in Frankreich vor der Fronde 1623-1648, Leipzig 1954; heftig kritisiert von Roland MOUSNIER, Fureurs paysannes. Les paysans dans les révoltes du XVII siècle, Paris 1967, sowie Yves-Marie BERCÉ, Crocquants et Nu-Pieds. Les soulèvements paysans en France du XVI $\mathrm{I}^{\mathrm{e}}$ au XIX $\mathrm{Xiècle}^{\mathrm{e}}$ Paris 1991 [zuerst 1971]; sowie Emmanuel LE ROY LADURIE, Le Carnaval de Romans. De la chandeleur au mercredi des Cendres 1579-1580, Paris 1979. Den Stand der Forschung faßt Jean NiCOLAS ( $\mathrm{Hg}$.), Mouvements populaires et Conscience sociale $\mathrm{XVI}^{e}-\mathrm{XIX}^{e}$ siècle, Paris 1985, zusammen. Wieder aufgegriffen wird die Frage der Revolten in französischen Städten der Frühneuzeit bei William BEIK, Urban Protest in Seventeenth-Century France. The culture of Retribution, Cambridge 1997. 
on ging von der Befürchtung, dem Spott der Bevölkerung ausgesetzt zu sein, bis zu einem Gefühl körperlicher Bedrohtheit. Hinzu kommt, daß insbesondere die aus dem übrigen Frankreich stammenden Richter am Parlament sich mit einem Provinzleben in Metz und vor allem in Toul kaum abgefunden hatten.

Geht man davon aus, daß der Großteil der Bevölkerung der neuen Situation aber grundsätzlich eher gleichgültig gegenuberstand und keinerlei Zeichen für bedrohliche Unruhen zeigt, muß der Grund für diese offenbar als wenig erfreulich empfundene Situation der königlichen Amtsträger vor Ort an anderer Stelle zu suchen sein. Er scheint am ehesten im problematischen Verhältnis der Verwaltung zum Militär zu liegen. Zwar hatte erst die militärische Besetzung die Grundlage für alle weiteren Entwicklungen im Sinne einer Eingliederung gelegt und zwar schützte und flankierte die Präsenz der Garnison jede Maßnahme der sich allmählich etablierenden königlichen Verwaltung. Auch waren gerade die ersten Justizbeamten, die von königlicher Seite in die TroisÉvêchés entsendet wurden, durch ihre Funktionen als Richter in Streitigkeiten zwischen Garnison und Bevölkerung ursprünglich mit wichtigen Funktionen für diesen notwendigen Ausgleich versehen. Doch trotz dieser funktionalen Unterstutzung waren die Konfliktlinien und Rivalitäten von Anfang an vorgegeben. Denn problematischer als die Durchsetzung gegenüber den untergeordneten Gerichten war für die königlichen Institutionen in Metz, Toul und Verdun die angesprochene Kontrollaufgabe über die in den Trois-Évêchés stationierten Truppen. Auch beim Parlament von Metz führte diese bald nach Arbeitsbeginn des Gerichts zum offenen Konflikt zwischen den Richtern und den Soldaten des Königs.

In den Schreiben der Amtsträger ist so eine deutliche Strategie erkennbar. Die Kritik an der Garnison oder gar dem Gouverneur war insbesondere in der Kriegssituation der 1630er Jahre problematisch. Das Argument, man müsse die noch widerstrebende Bevőlkerung durch die Justiz für den König gewinnen, bot den Justizbeamten hingegen zahlreiche gute Gründe für eigene Forderungen. Teilweise wurde eine direkte Verbindung zwischen der materiellen Ausstattung der Amtsträger und dem Ansehen königlicher Institutionen allgemein gezogen: Nur wenn die Männer des Königs ihren Mitbürgern finanziell überlegen waren, wurde argumentiert, konnten sie dessen Interessen auch gegenüber der kritisch beobachtenden Bevölkerung durchsetzen.

Am Fallbeispiel der Trois-Évêchés wird damit sehr gut deutlich, wie die Akteure in einem politischen Feld interagieren (konnten). Denn das Spannungsfeld der Akteure im Diskurs um die Gerichtsbarkeit legt Schwierigkeiten und Möglichkeiten bei der Eingliederung ins Königreich, aber auch grundsätzliche Schwierigkeiten und Chancen frühneuzeitlicher Herrschaft deutlich offen. Dieses Spannungsfeld kann trotz vieler Überschneidungen und Unschärfen als 
eine Art Dreieck dargestellt werden, innerhalb dessen in den Trois-Évêchés kommuniziert wird ${ }^{11}$. An der Spitze hat sich der König etabliert, während an den beiden nebeneinander gelegenen Enden die königlichen Amtsträger auf der einen und die Bewohner, allen voran ihre Bischöfe und Stadtregierungen auf der anderen Seite, zu finden sind. Hierbei ging die positive Bezugnahme eines Akteurs auf einen zweiten immer mit der Herabsetzung des dritten einher.

Positiver Bezugspunkt war meist der König, der aus diesem Grund an der Spitze des Dreiecks steht. Wenn sich Bewohner und Amtsträger an den König wandten, meinten sie damit allerdings weniger die konkrete Person als vielmehr die letztlich entpersonalisierte Figur des Herrschers. Seine Position, sein Ansehen und auch das seiner Pariser Institutionen (königlicher Rat und Parlament) scheint relativ schnell nach 1552 und weitgehend unangefochten einen wesentlich bedeutenderen Platz in Metz, Toul und Verdun eingenommen zu haben als es dem Kaiser je gelungen war.

Hilfreich war hierbei die Rechtskonstruktion der Protektion, die auf in den Trois-Évêchés bereits bekannten Elementen basierte. Hiermit, sowie durch deren allmähliche Ausweitung auf die Souveränitätsidee, konnte ein bei anderen Herrschaftswechseln häufig erkennbarer "Bruch» vermieden werden. Ähnlich ist die beständige Garantie und feierliche Bestätigung der städtischen und bischöflichen Privilegien durch den König zu werten, die die kaiserliche Praxis äußerlich fortsetzte.

Letztlich war aber in den Trois-Évêchés entscheidend, wer sich als Garant der Privilegien darstellen konnte. Zudem bot die Zeremonie als solche einen idealen Anla $B$, mit den Vertretern der Städte in Verhandlung zu treten, denn wenn die Privilegien der Bestätigung des Königs bedurften, waren auch alle übrigen Fragen der städtischen Verwaltung Verhandlungsmasse. Deutlich wird dies beispielsweise bei der Durchsetzung der königlichen Gerichtsbarkeit in den cas royaux in Toul und Verdun um die Jahrhundertswende, die mit einer grundsätzlichen Bestätigung der städtischen und bischöflichen Gerichtsrechte einherging. Die Garantie der Privilegien bot dem Herrscher damit eine Art Trojanisches Pferd, mit dem Herrschaftsansprüche durchgesetzt werden konn$\operatorname{ten}^{12}$.

Die Bischöfe, Domkapitel, Magistrate und Bewohner (vor allem der Städte) machten schon durch die Anzahl und Häufigkeit ihrer Suppliken und Re-

"Ebenfalls in einem geometrischen Bild stellt Werner NÄF, Die Epochen der neueren Geschichte. Staat und Staatengemeinschaft vom Ausgang des Mittelalters bis zur Neuzeit, 2. Aufl., Aarau 1959/60, Bd. 1, S. 432, das Spannungsverhältnis innerhalb des frühneuzeitlichen Staates dar: Er wählt die Elipse, deren einer Brennpunkt die Stände, der andere der Fürst ist.

${ }^{12}$ Dies zeigt auch am Beispiel der taille HICKEY, Coming of French Absolutism, v.a. S. 161-191. 
monstrationen deutlich, da $\$$ sie und ihre Interessen nicht ignoriert werden konnten. Wie Beat Hodler in seiner Untersuchung zu »Doléances, Requêtes und Ordonannces « für das 16. Jahrhundert gezeigt hat, war der König geradezu verpflichtet, auf diese Form der Partizipation einzugehen ${ }^{13}$. Gleichzeitig zeigt jedoch auch hier die Tatsache, daß sich die Stände der Trois-Évêchés nach Paris und nicht mehr an Kaiser und Reichstag wandten, um ihre Interessen durchzusetzen, eine gewisse Anerkennung der königlichen Oberherrschaft ${ }^{14}$.

Unter diesem Gesichtspunkt stabilisierte sich das politische System in den Trois-Évêchés nicht trotz, sondern wegen eines gewissen Maßes an Kritik allmählich selbst. So machten die Bewohner, wenn sie sich gegen Maßnahmen der königlichen Amtsträger vor Ort an den König wandten, gleichzeitig deutlich, daß sie diesen als obersten Garanten der Gerechtigkeit akzeptierten. Allerdings nahmen sie den Herrscher damit auch in die Pflicht, ihnen gegenüber den Amtsträgern oder bestimmen Vorhaben im Namen der Gerechtigkeit Zugeständnisse einzuräumen.

Das gleiche gilt für die Amtsträger: Wendeten sie sich mit einem gewissen Gefühl der Bedrohung durch die Bevölkerung an den König, betonten sie erneut das starke Band zwischen ihnen und dem Herrscher, der ihnen die Ausübung ihres Amtes ermöglichte. Gleichzeitig machten sie deutlich, daß das Ansehen des Herrschers direkt davon abhing, wie sie als seine Vertreter vor Ort finanziell gestellt waren und welche Kompetenzen ihnen gegeben wurden.

Und schließlich war der König ebenfalls auf eine gewisse Zustimmung in der Bevölkerung und bei seinen Amtsträgern angewiesen und machte Zugeständnisse, um neben dem Land eben auch die Herzen zu erobern, wie dies Richelieus Vertrauter Abel Servien in Worte gefaßt hatte.

Auch wenn Selbst- wie Fremdbezeichnungen der Bewohner im Diskurs um die französische Rechtspolitik deutlich machen, daß der Begriff des "Französischwerdens « für die Ereignisse in den Trois-Évêchés nach 1552 in vielerlei Hinsicht nicht geeignet scheint, da er ein »Nationalgefuhl« bei den Bewohnern der Trois-Évêchés suggeriert, das so sicherlich nicht zu beobachten ist, so läßt sich doch eine deutliche Verschiebung der Referenzpunkte erkennen. Denn während bei den Amtsträgern noch lange die deutliche Trennung in "Wir« (français) und »Ihr « (lorrains oder wertfrei: ceux de ce pays) ${ }^{15}$ vorherrschte, zeigte sich nach 1633 innerhalb der Städte etwa bei der Errichtung der königlichen bailliages, wie sich die Bezugspunkte der Argumentation in Metz von

\footnotetext{
${ }^{13}$ HODLER, Doléances, v.a. S. $64-67$.

${ }^{14}$ In dem Moment, in dem ein Akteur kommuniziert, akzeptiert er auch das dieser Kommunikation zugrundeliegende Regelsystem. Vgl. dazu GIDDENS, Konstitution der Gesellschaft, v.a. S. 387-389.

${ }^{15}$ Vgl. dazu KOSELLECK, Zur historisch-politischen Semantik, S. $211 \mathrm{f}$.
} 
den Privilegien einer Reichsstadt $\mathrm{zu}$ denen einer bonne ville de France verschoben hatten.

Geht man wie in der vorliegenden Arbeit von den Kommunikationsabläufen aus, die sich hier am Beispiel der Rechtspolitik herausarbeiten lassen, erscheint die Etablierung moderner Staatlichkeit damit weder als zwanghaftes "Fortschreiten«, noch als Mißerfolg, sondern vielmehr als etwas, das sich in und durch seine ständige Diskussion formte ${ }^{16}$. Erst dieses komplizierte Gleichgewicht, in dem jede Maßnahme zwischen König, Bevölkerung und Amtsträgern abgestimmt werden mußte, machte die Eingliederung langsam, aber ohne größere Widerstände möglich. Statt Disziplinierung oder »Französisierung « schien das Ziel der königlichen Politik in den Trois-Évêchés eher Legitimität und Ausgleich zu sein. Dem in einer Denkschrift formulierten Vorhaben, aus den Lothringern sujets $d u$ Roi zu machen, stand das weit häufiger genannte Postulat, ihre Herzen zu gewinnen, lange Zeit im Wege. Dies hatte auch der Hofhistoriker Godefroy erkannt, der am Rand der von ihm gesammelten Briefe aus Metz zur Grußformel anmerkte: Nota: ils ne mettent poinct "subiects " 17 .

\footnotetext{
${ }^{16} \mathrm{Vgl}$. ReINHARD, Geschichte der Staatsgewalt, S. 22.

${ }^{17}$ Institut, Godefroy, 257.
} 
